\title{
Impacto de un Sistema Web para Optimizar Insumos en Negocio de Comida
}

\author{
Impact of a Web System to Optimize Food Business \\ Supplies
}

Vilma Álvarez-Intriago ${ }^{1,}{ }^{*}$, Fernando Torres-Samaniego ${ }^{1}$

INFORMACIÓN DEL

ARTÍCULO

Fecha de recepción: 6 de enero de 2019.

Fecha de aceptación: 27 de febrero de 2019

1 Universidad de Especialidades Espíritu Santo, Facultad de Sistemas, Telecomunicaciones y Electrónica, Km. 2.5 Vía la Puntilla, Samborondón, Ecuador.

* Autor de correspondencia:

Vilma Álvarez Intriago, Universidad de Especialidades Espíritu Santo, Facultad de Sistemas, Telecomunicaciones y Electrónica, Km. 2.5 Vía la Puntilla, Samborondón, Ecuador

E-mail: vkalvarez@uees.edu.ec

Tlf.: (593-4) 283-5630 ext. 514

ENLACE DOI

http://dx.doi.org/10.31095/investigatio 2019.12.7

\begin{abstract}
Resumen
Los avances tecnológicos han contribuido a que las empresas alcancen ventajas competitivas, tal como, la implementación de sistemas informáticos, los cuales aportan y cubren falencias de procesos de información. Si se habla de negocios de comida, uno de los principales objetivos es la gestión de los insumos y su inventario, con los cuales se busca reducir costos sin afectar la calidad de los productos. Por lo anteriormente expuesto, éste artículo tiene como objetivo analizar el impacto de una aplicación web desarrollada con metodología ágil Scrum, enfocándose en la optimización de los insumos de un local de comida. Para lograr esto, se consideraron tres etapas para la implementación: definición del producto, diseño de la solución y el desarrollo de la aplicación web; obteniendo como resultado, un sistema informático que permite mejorar el control de los inventarios; así como la planificación de adquisición y distribución de los insumos.
\end{abstract}

\section{Palabras Clave:}

Aplicaciones web; insumos; inventario; scrum; sistemas de información. Clasificación JEL: C61.

\section{Abstract}

Technological advances have contributed to companies reaching competitive advantages, such as the computer system implementation that will cover information process shortages. One of the objectives of the food business is to manage supplies and their inventories, in order to achieve cost reduction without affecting product quality. This article, therefore, purports to analyze the impact of a web application developed with agile Scrum methodology focused on a restaurant's supply optimization. To achieve this, three implementation stages were considered: product definition, design solution and web application development; as a result, a computer system that allows inventory control was improvement; it also allows input acquisition, distribution planning and inventory control.

\section{Keywords:}

Information systems; inputs; inventory; Scrum; web application.

JEL Classification: C61.

103 


\section{Introducción}

El rápido crecimiento de las nuevas tecnologías ha hecho que la economía mundial la identifique como un aliado estratégico para el control y la toma de decisiones en los negocios, ya que proporciona información clave para definir objetivos propuestos. En el caso de la industria gastronómica, las medianas y grandes empresas generalmente aprovechan los beneficios de la tecnología mediante la implementación de un software que cumpla con las necesidades principales del giro del negocio; es decir, de sistemas informáticos acoplados al modelo del negocio implantado. Tal como asegura Trasobares (2003) uno de los objetivos principales de los sistemas es apoyar los objetivos y las estrategias de los negocios. Así es, como varias empresas han documentado su ventaja competitiva adoptando sistemas de información, a pesar que no todas obtienen los mismos resultados (Andreu, Ricart, y Valor, 1991). De acuerdo a las necesidades de cada entidad del negocio, se requieren diferentes tipos de sistemas de información que cubrirán la necesidad de acuerdo a los objetivos del negocio (García-Holgado y García-Peñalvo, 2015).

En el mercado existen varias herramientas que satisfacen las necesidades básicas de estas empresas, pero que poseen desventajas y/o limitaciones, ya que no permiten controlar todos los aspectos que conlleva el movimiento del negocio. Gutiérrez y Vidal (2008) menciona que uno de los problemas típicos de los negocios de comida es la existencia de los excesos y/o faltantes de insumos en sus inventarios.
Sobre esto, Osorio (2008) explica que el control de inventarios es uno de los temas más complejos en logística y gestión de la cadena de abastecimiento, ya que se debe gestionar que los locales de comida no tengan exceso o falta de insumos en sus bodegas para cumplir con sus operaciones diarias.

Rossetti y Arcusín (2013) aclaran que la finalidad de una buena gestion de los inventarios es encontrar el equilibro entre el costo del mantenimiento y el stock que se posee mencionado inventario. Es por esto, que es considerada una actividad transversal de vital importancia en las cadenas de abastecimiento, las cuales constituyen uno de los procesos logísticos más complejos de administrar (Gutiérrez y Vidal, 2008). Uno de sus principales problemas es el poseer, en inventario, un exceso de lo que se vende poco $\mathrm{y}$, menos productos de los que tienen mayor venta. Esto se debe a la falta de información exacta y oportuna sobre la demanda que se genera en las ubicaciones de los negocios. Consecuentemente, se debe realizar un correcto manejo del inventario, involucrando todos los sectores de la empresa, en la cual se implementan desde políticas hasta el análisis de necesidad de abastecimiento, de acuerdo a las estadísticas que se poseen con las operaciones del negocio (Rossetti y Arcusín, 2013). Así lo corrobora Sánchez, Vargas, Reyes y Vidal (2011) al mencionar que poseer un sistema de costos permite tener efectivo control del inventario, a fin de mantener una coordinación y cooperación entre los elementos del sistema. Su principal objetivo es reclasificar las cargas o costos 
para brindar una fuente de información eficaz y así ayudar a la toma de decisiones de cada departamento (García, Marín y Martinez, 2006). Además, se relaciona con el núcleo de operaciones de la empresa, en donde se realizan todas las actividades para convertir los insumos en los bienes y/o servicios que se brindarán al usuario (Chacón, 2007).

Según González y Domínguez (2012), un sistema informático de costos que se controla de manera eficaz y eficiente es aquel que proporciona información precisa al negocio en forma de reportes, con el cual se realiza el análisis de la información y, así conocer si los insumos se encuentran dentro de los parámetros establecidos y que facilita el proceso de toma de decisiones de acuerdo a la información brindada (Lavolpe, 2005).

Sin embargo, es importante que tanto el sistema de inventario como el de costos sean aplicaciones web que sean accedidas desde cualquier parte a fin de que el seguimiento y control sea globalizado. Así lo dice Arbeláez, Medina y Chaves (2011), quienes consideran que las aplicaciones web son sistemas de software que necesitan un lenguaje de programación diseñado para realizar páginas web, PHP, acrónimo recursivo de HyperText Preprocessor o Preprocesador de Hipertexto, el cual es de código abierto y diseñado para realizar páginas web dinámicas. Se llaman páginas web dinámicas a las que poseen contenido variable, normalmente debido a que está vinculado a una base de datos de donde se obtiene la información (Blandón y López, 2012).
Una de las ventajas más importantes de este lenguaje es que no existe la necesidad de una herramienta específica para el desarrollo, sino que se pueden utilizar diversas aplicaciones para editar los archivos, desde un block de notas hasta un programa desarrollado por un tercero con características adicionales (López, 2011). Esto lo hace una elección apropiada al momento de elegir un lenguaje de programación para proyectos sencillos (Avello, Pérez y Gayo, 2001), complementando se utiliza JavaScript asíncrono y XML (AJAX, por sus siglas en inglés). De acuerdo a Bazán (2008), éstas son un grupo de técnicas que se ejecutan en los navegadores para el desarrollo de las aplicaciones web. Una de las principales funciones es recuperar información de manera asincrónica, procesándola en segundo plano para poder visualizarla en la pantalla sin tener que refrescar la página web, mejorando los tiempos de respuestas y la funcionalidad del sistema (Garrett, 2005).

Actualmente, el lenguaje de programación PHP, es usado por millones de sitios web, desde pequeñas hasta grandes empresas, incluyendo a Facebook, Wikipedia y WordPress. A partir de la versión 5, se introdujo un modelo más complejo; entre las características añadidas se encuentran el manejo de clases abstractas, clonación, manejo de excepciones, tipos sugeridos, entre otros (García, 2005). Por otro lado, una de las alternativas para mantener la información de los sistemas en un almacenamiento seguro y poder acceder a ella en todo momento, es migrar los sistemas a la nube, la cual permite 
mejorar el rendimiento gracias a los servidores que procesan las aplicaciones. Según a Armbrust, et al. (2010) la computación en la nube se refiere a las aplicaciones que son entregadas como servicios en internet, al hardware y aplicaciones en los centros de datos que brindan el servicio y que ofrecen beneficios para fidelizar a los clientes y tener la información necesaria en cualquier momento y en cualquier lugar.

La preocupación de las empresas de migrar a la computación en la nube se debe a que, al contratar los servicios de un tercero, estas no conocen con certeza la ubicación física de su información ni las políticas de seguridad implementadas, por lo que los negocios aún analizan si es necesario o no migrar sus sistemas a la nube (Aruquipa, 2012). La importancia de esta tecnología es que se puede acceder al contenido desde cualquier dispositivo con acceso a internet y por varios usuarios simultáneos, un aspecto muy útil en el ámbito educativo, laboral y personal (Franco, Trejo y Román, 2016).

Así, el Consorcio W3C define los servicios web como sistemas diseñados para soportar la interoperabilidad entre distintos sistemas de información que se encuentran en una misma red. Estos servicios definen mecanismos estandarizados para la descripción, localización y comunicación con distintas aplicaciones en línea (Marcos, De Castro y Vela , 2004). Una de las grandes ventajas de los servicios web es que permite que los sistemas sean independientes de las plataformas para las que fueron desarrollados o donde se encuentren instalados, debido al uso de estándares abiertos para la comunicación como Lenguaje de Marcas Extensible (XML), Lenguaje de Marcas de Hipertexto (HTML) y Protocolo para Transferencia Simple de Correo (SMTP) (Losavio, Matteo y Rahamut, 2006). Además, facilitan la integración de los servicios y software de distintas empresas que pueden estar alojadas en diferentes lugares, haciendo posible el funcionamiento en conjunto en el intercambio de información (Santamaría, Ballesteros y González, 2015)

Por lo anteriormente expuesto, el objetivo de este artículo es desarrollar una aplicación web centralizada que permita controlar el inventario y gestionar los insumos de un restaurante con el fin de reducir costos, evitar pérdidas de insumos y facilitar la distribución de los mismos entre los locales del negocio. Para esto, primeramente, se identifican los procesos involucrados en el control de inventario y la gestión de consumo; luego se establece el diseño y la arquitectura de los mismos; finalmente, se procede a desarrollar la aplicación web, realizando validaciones y pruebas del correspondiente funcionamiento.

\section{Materiales y métodos}

Para el desarrollo de la investigación se establecieron tres etapas: la primera es la identificación de los procesos involucrados en donde se realiza la definición del producto; la segunda comprende el diseño y arquitectura de la solución; la tercera, el desarrollo de la aplicación involucrando las pruebas y puesta a producción.

106 
Identificación de procesos involucrados

En esta etapa, se recolectó toda la información y procesos relacionados a los requerimientos del restaurante por medio de entrevistas a empleados y a los dueños. En base a esto, se definieron las características de la aplicación web que comprende el control de inventario y la gestión de consumo.

\section{Diseño y arquitectura del programa}

Para esta segunda etapa, se utilizó un marco de trabajo ágil llamado metodología Scrum; el cual es considerado un framework que se basa en principios ágiles para la gestión de desarrollos complejos (Nazareno, Leone y Gonnet, 2013). Yazyi (2011) afirma que Scrum está basado en ciclos cortos y se enfoca en la producción de valor, la mejora continua del proceso y orientación a las personas. Así también, Huiza (2012) menciona que esta metodología es flexible y que es la adecuada por el dinamismo en los requerimientos del cliente y los cambios en las exigencias del desarrollo.

\section{Desarrollo de la aplicación web}

En la última, etapa se desarrolló el aplicativo de acuerdo al enfoque que se detalló en la etapa de diseño, utilizando el lenguaje de programación PHP, bajo un cronograma planificado. Así, de acuerdo a la metodología expuesta anteriormente, cada día se realizaron reuniones con el usuario para revisar el avance del desarrollo, de acuerdo a prioridades que ya han sido establecidas. En la Figura 1 se detalla la forma en la que se manejan los ciclos con Scrum (Colla, 2012).

En este desarrollo se manejan roles que cumplirán un papel importante en cada fase de implementación del

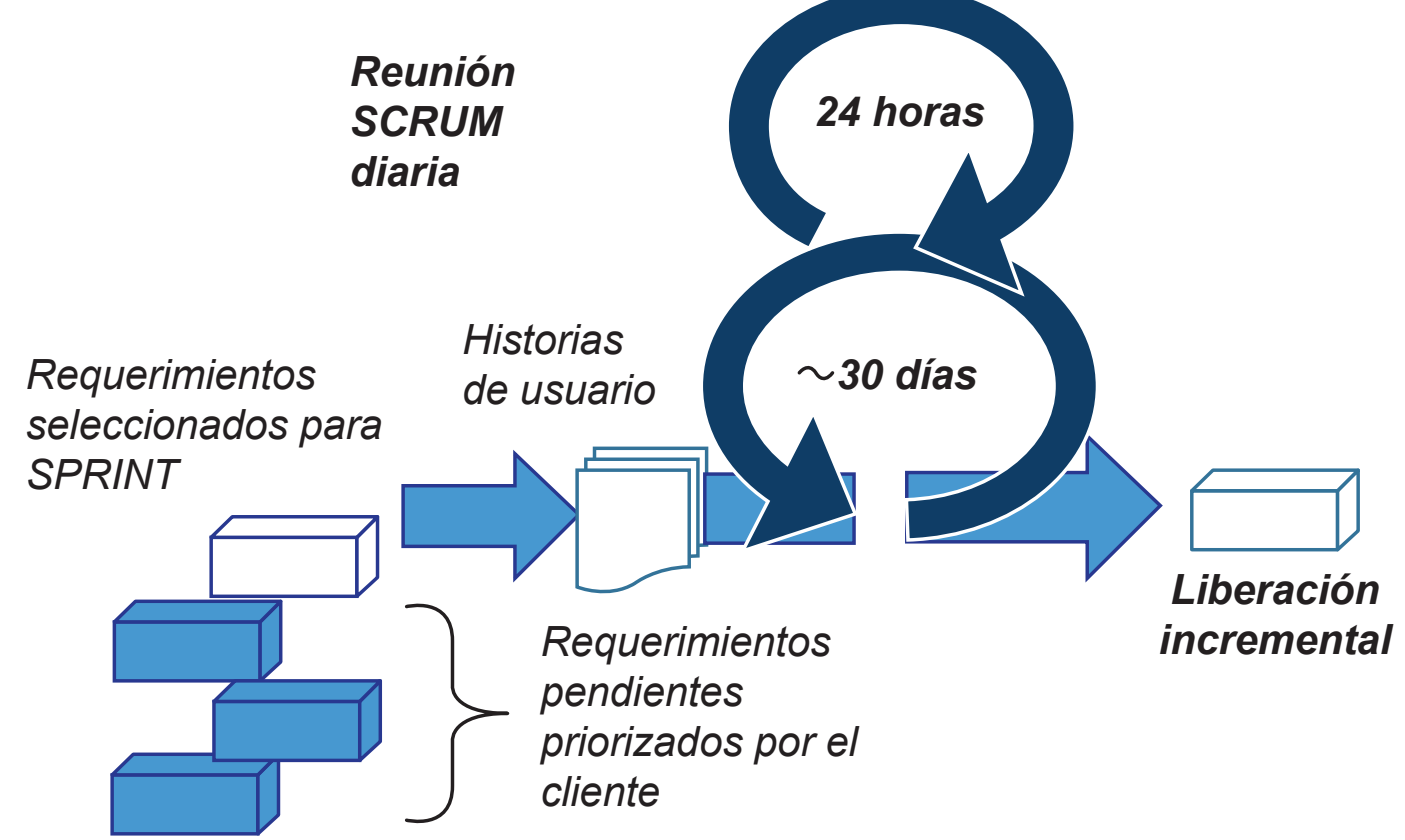

Figura 1. Diagrama de la descripción conceptual de un ciclo Scrum. de desarrollo de software usando la metodología Scrum. Tomado de "Marco para evaluar el valor en metodología Scrum", por Colla (2012). 
Tabla 1.

Actividades de la definición del producto.

\begin{tabular}{lll}
\hline \multicolumn{1}{c}{ Actividades } & \multicolumn{1}{c}{ Entrada } & \multicolumn{1}{c}{ Salida } \\
\hline $\begin{array}{l}\text { Definición del proceso } \\
\text { operativo actual }\end{array}$ & $\begin{array}{l}\text { Información de las características del } \\
\text { sistema que poseen actualmente }\end{array}$ & $\begin{array}{l}\text { Definición de las especificaciones del } \\
\text { sistema actual para la operatividad }\end{array}$ \\
$\begin{array}{l}\text { Definición de los problemas } \\
\text { dentro del proceso actual }\end{array}$ & $\begin{array}{l}\text { Entrevistas para conocer los problemas as } \\
\text { actuales }\end{array}$ & $\begin{array}{l}\text { Ficha de los requerimientos y/o } \\
\text { problemas que se pretenden solucionar }\end{array}$ \\
$\begin{array}{l}\text { Análisis de impacto de la } \\
\text { solución con respecto al negocio }\end{array}$ & $\begin{array}{l}\text { Información de las características y } \\
\text { módulos que se desarrollarán }\end{array}$ & $\begin{array}{l}\text { Ficha de tareas de los módulos que } \\
\text { afectarán al sistema }\end{array}$ \\
$\begin{array}{l}\text { Definición de la solución } \\
\text { tomando en consideración el } \\
\text { problema actual }\end{array}$ & $\begin{array}{l}\text { Información que cubra todos los } \\
\text { requerimientos del cliente }\end{array}$ & $\begin{array}{l}\text { Definición de especificaciones del } \\
\text { sistema que se desarrollará }\end{array}$ \\
\hline
\end{tabular}

producto (Orjuela y Rojas, 2008). Uno de los roles más importantes es el denominado "propietario del producto", el cual tendrá una participación constante en el desarrollo del mismo y será el encargado de transmitir la idea y los aspectos que se requieren para el producto final (Yazyi, 2011). Así, para cumplir con los tiempos y los resultados esperados, el equipo deberá tener un alto grado de autonomía y responsabilidad, alineándose a lo que el propietario del producto espera sobre cada entregable (Barrios, et al., 2011). Por otro lado, ya que la metodología de desarrollo Scrum maneja implementaciones de software cortos o entregables, llamados sprints; en ésta investigación, se determinaron cuatro sprints, El Sprint 1 se enfoca en la administración de usuarios, perfiles y otorgación de los accesos de acuerdo al tipo de usuario; el sprint 2 consiste en administrar los catálogos principales que interactúen con la operatividad del negocio, dentro de los cuales se encuentran las recetas, pre-recetas, $\mathrm{y}$ categorías de los platos. Luego, el sprint 3 se encamina en la administración de los insumos, la de inventarios, el traslado de insumos del inventario de un local a otro, y la carga manual de la venta para la disminución de los insumos. Por último, el sprint 4 se dedicó a la reportería del aplicativo web, en el cual se generaron archivos con extensión .pdf.

\section{Resultados}

Procesos involucrados en el control de inventario y la gestión de consumo

En la primera etapa del proyecto, los procesos involucrados permitieron definir el diseño de la aplicación web y posteriormente su desarrollo. Con ese propósito, en la Tabla 1 se detallan las actividades que se realizaron para identificar mencionados procesos.

Así, con los procesos identificados, se determinaron las características por módulo; las mismas que se muestran en la Tabla 2.

Tabla 2.

Características por Módulo.

\begin{tabular}{cl}
\hline Módulo & \multicolumn{1}{c}{ Característica } \\
\hline Facturación & Mesas para tomar pedido de ventas \\
Facturación & Facturación de cuentas \\
Servicios & Opción para entrega a domicilio \\
Caja & Entrada y salida de dinero en caja \\
Reportería & Reporte de ventas y de insumos \\
Inventario & Manejo básico de inventario \\
\hline
\end{tabular}


Como resultado de reuniones se estableció que el manejo de inventario era uno de los problemas más importantes que se resolvían ya que el sistema actual solo manejaba un inventario básico para la administración de las recetas. Sin embargo, como las recetas y las pre-recetas se componen de uno o varios insumos, al momento de modificar los insumos o la cantidad usada en una pre-receta, se deberá modificar todas las recetas que la esté usando el negocio, administrando los productos finales tal como lo muestra la Figura 2:

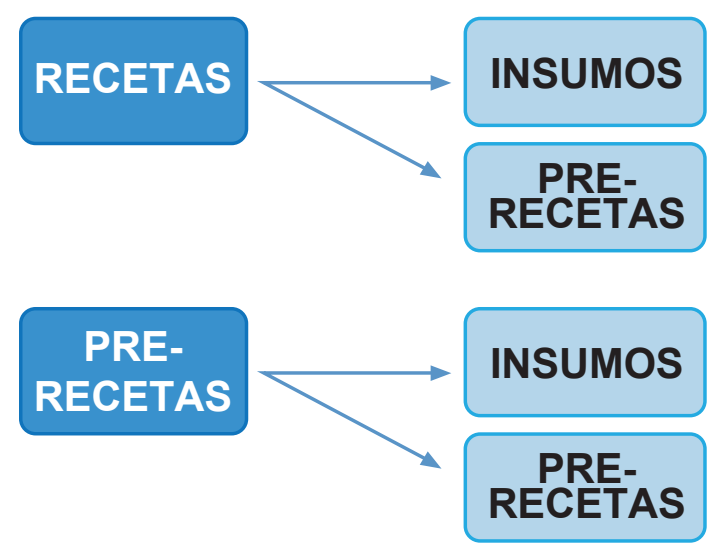

Figura 2. Administración de recetas y pre-recetas en la aplicación web.

En base a esto, se obtuvieron todos los

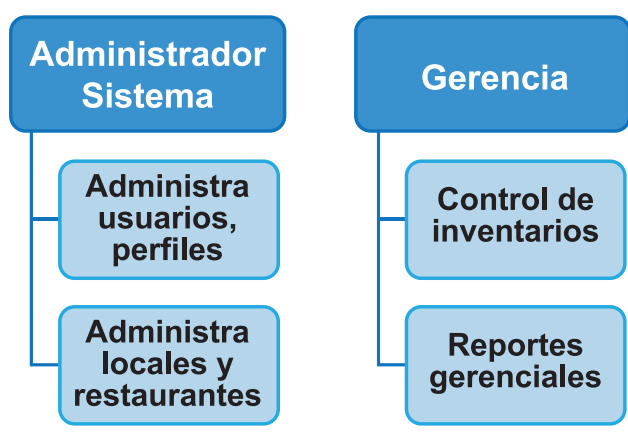

Figura 3. Perfiles del sistema web. requerimientos de los clientes, llamados "historias del usuario", y en el desarrollo se los agruparon por su prioridad; las cuales se detallan en la Tabla 3:

Tabla 3.

Definición de las historias del usuario.

\begin{tabular}{|c|c|c|}
\hline Número & Problema & Prioridad \\
\hline 1 & $\begin{array}{l}\text { Visualización unificada de los } \\
\text { inventarios entre locales }\end{array}$ & ALTA \\
\hline 2 & Alarma de falta de stock & MEDIA \\
\hline 3 & Cargar recetas y pre-recetas & ALTA \\
\hline 4 & $\begin{array}{l}\text { Reporte de stock actual en inventario } \\
\text { normal y de producción }\end{array}$ & ALTA \\
\hline 5 & Reporte de recetas vendidas & ALTA \\
\hline 6 & Creación de nuevas recetas & ALTA \\
\hline 7 & $\begin{array}{l}\text { División entre inventario normal y el } \\
\text { inventario de producción }\end{array}$ & ALTA \\
\hline 8 & $\begin{array}{l}\text { Sugerencia de precio de la receta de } \\
\text { acuerdo al costo total de los insumos }\end{array}$ & ALTA \\
\hline
\end{tabular}

\section{Diseño y arquitectura de la solución}

De reuniones con la Gerencia General se obtuvo el diseño de la solución, cubriendo todos los requerimientos ya solicitados por el usuario, y a la vez definiendo cuatro perfiles para accesos al sistema: Administrador del Sistema, Gerencia, Jefe de Costos y Administrador del local junto con las opciones autorizadas para los mismos. Los cuales se muestran en la Figura 3:
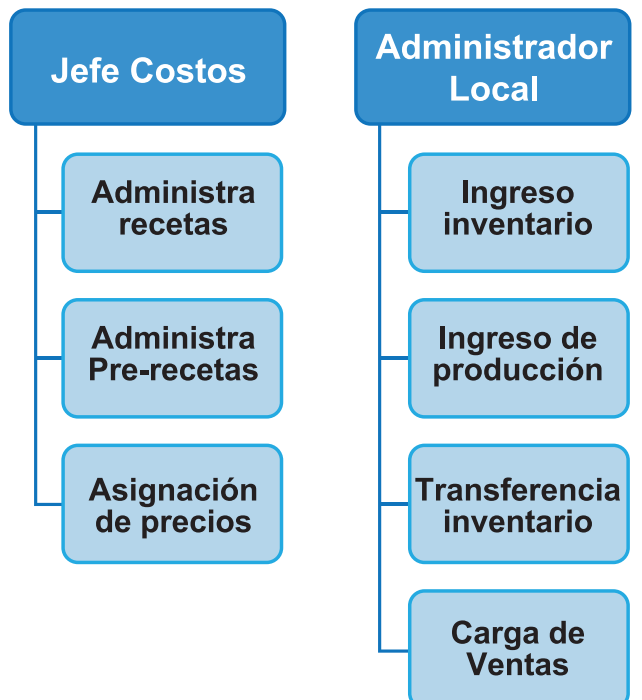
Otro resultado adquirido fue la determinación de las opciones principales de la solución: administración de recetas, administración de pre-recetas, asignación de precios a insumos, asignación de precios a recetas, emisión de ventas, ingreso de inventario, ingreso de producción, control de venta, control de inventario y producción; las cuales fueron implementadas con el lenguaje de programación PHP en base a un análisis de acoplamiento con la metodología ágil de software llamada Scrum, caracterizándose principalmente por ser un proceso interactivo e incremental mediante Sprints, gracias al cual el equipo de desarrollo y los usuarios trabajaron en conjunto. En la Tabla 4 se detalla los Sprints utilizados en el desarrollo.

Tabla 4.

Sprints en el desarrollo de la solución.

\begin{tabular}{|c|c|c|}
\hline $\begin{array}{l}\text { Número de } \\
\text { Sprint }\end{array}$ & Duración & Resultado \\
\hline 1 & 3 semanas & $\begin{array}{l}\text { Desarrollo del módulo de seguridad y } \\
\text { accesos al sistema }\end{array}$ \\
\hline 2 & 3 semanas & $\begin{array}{l}\text { Desarrollo de los módulos de } \\
\text { parametrización de catálogos del sistema }\end{array}$ \\
\hline 3 & 4 semanas & $\begin{array}{l}\text { Desarrollo de los módulos de carga de } \\
\text { venta, aumento y disminución de inventario }\end{array}$ \\
\hline 4 & 2 semanas & $\begin{array}{l}\text { Desarrollo de la reportería necesaria para } \\
\text { visualizar la información }\end{array}$ \\
\hline
\end{tabular}

Se obtuvieron las tareas de cada Sprint al igual que las horas que podían tomar cada uno, pudiendo realizar la planificación de los tiempos, midiendo el cumplimiento y teniendo una retrospectiva del Sprint realizado. Como resultado del desarrollo del Sprint 1 se pudo iniciar sesión en el aplicativo y visualizar las opciones de acuerdo al perfil que fue asignado al usuario. Así también, con la implementación del Sprint 2, se lograron visualizar los productos finales del negocio, calcular los costos, márgenes de ganancia. Esto también permitió conocer con exactitud los insumos que se disminuirían cuando se envíe una receta a producción. Luego, como resultado del Sprint 3, se consiguió realizar un control de los insumos y sus movimientos. Adicionalmente, con la carga de las ventas de los locales, se disminuyó automáticamente los insumos de acuerdo a las recetas. Finalmente, consecuentemente de la implementación del Sprint 4 se obtuvo la reportería del aplicativo web, en el cual se generaron archivos con extensión .pdf.

\section{Validaciones y pruebas}

Por otro lado, la puesta en producción del sistema aportó con una estandarización total de las recetas y pre-recetas que maneja el restaurante. Las pruebas que se realizaron por cada Sprint que fue entregado resultaron exitosas. Se validó que todos los requerimientos que el cliente solicitaba se cumplan a cabalidad $y$ funcionen de manera adecuada, tanto visualmente como en la integridad de datos almacenados en la base. Las pruebas ayudaron a conocer de una mejor manera la operatividad del negocio por lo cual si el usuario deseaba mejoras o nuevos módulos resultaría más fácil de integrarlo al desarrollo ya realizado.

Con la visualización de márgenes de ganancia de las recetas y el cálculo correcto de los insumos se pudo gestionar de una manera más precisa el uso de insumos, como también el cálculo del stock que se encontraba en el inventario 
normal como también en el inventario de producción.

Para finalizar, la carga manual de ventas ayudó a mantener los indicadores de los inventarios actualizados con el fin de planificar las nuevas adquisiciones de los insumos en los locales del restaurante.

\section{Discusión}

El desarrollo e implementación de una aplicación web centralizada administrando los insumos, recetas, y pre-recetas del restaurante permite el control de stock y márgenes de los precios por cada local del restaurante; incrementando la utilidad en las ventas, así como la disminución de la pérdida de insumos por caducidad, calidad o un manejo deficiente; ya que para evitar costos innecesarios $y$ potenciar el uso de los recursos de la empresa, lo ideal es identificar de manera precisa la necesidad de los insumos requeridos en los procesos de producción de cada sucursal.

Según la Asociación Española de Contabilidad y Administración de Empresas (2013), el acceso en tiempo real a la información en toda la organización tiene sinergias positivas, dado que se reducen tareas duplicadas, se dispone de más tiempo adicional y favorece una respuesta rápida en la toma de las decisiones que lo requieran. Así, el uso de sistemas informáticos está cumpliendo un papel importante en el giro de los negocios; en el caso específico de la industria gastronómica, la administración de los insumos se ha convertido en una gestión clave, ya que permite optimizar la adquisición y planificación en el uso de los mismos. La presente investigación contribuyó en la mejora del manejo y estandarización de las recetas, en donde se evidencia una optimización en el uso de los insumos, como también un mejor control de los inventarios que poseen los restaurantes.

Benvenuto (2006) adiciona los efectos inmediatos y prácticos que tendrá la implantación de un sistema de información sobre las personas implicadas tales como: reducción y eliminación de tareas debido a la integración y automatización de funciones, se suma la reducción de uso de papel. Así, el desarrollo e implementación de un sistema web para el control de inventario y gestión de insumos en el restaurante, permite a la empresa evitar descuadres en el inventario, prevenir el excedente o falta de insumos, calcular los costos de producción y gestionar los procesos de planificación, adquisición y distribución de insumos para que, de esta manera, se minimicen los costos.

Por otra parte, el centro de excelencia de software libre (2011) detalla que un sistema de información implementado para un distribuidor de repuestos denominado Sifam España S.L, sistematizó todo el flujo de información en un único entorno, así también permitió obtener información para poder tomar mejores decisiones. Así también, el uso de una aplicación web permitió mejorar la manera en que visualiza y gestiona la información del restaurante de una forma unificada, brindando el acceso desde 
cualquier momento y en cualquier lugar a fin de considerar información relevante en la toma de decisiones.

EDICOM (2012) refiere al caso de aplicación exitosa de sistemas de información para el grupo Unilever, donde los beneficios obtenidos están en la mejora de las relaciones con el cliente, la optimización de procesos de adquisiciones y ventas, entre otros. Asimismo, Solmicro (2011) describe otra implementación satisfactoria de un sistema de información para la empresa metalmecánica mexicana Garsán, denominada Solmicro -eXpertis la cuál reemplaza al viejo sistema de información basado en AS400, permitiendo mejorar la productividad de todos sus departamentos, especialmente el financiero.

Por otro lado, al considerar Scrum como metodología de desarrollo ágil, en lugar de otra metodología, permitió planificar las fases para el desarrollo del sistema, enfocando la solución de acuerdo a las necesidades del negocio por medio de las reuniones periódicas realizadas con los usuarios claves (Alfonso, Mariño, y Godoy, 2011). Así también, las pruebas de los Sprints facilitaron corregir y mejorar las opciones entregadas de acuerdo a las recomendaciones realizadas por el usuario. En cuanto las pruebas de la aplicación que se realizaron en cada Sprint, se pudo observar el funcionamiento de las opciones desarrolladas; corrigiendo y mejorándolas cuando se presentaban novedades. Con la implementación de la metodología Scrum para el desarrollo de la solución, las reuniones diarias y la retroalimentación que se dieron con el cliente, permitieron cumplir los tiempos establecidos, garantizando que el desarrollo de la solución fuera enfocado hacia las necesidades y prioridades del mismo (Navarro, Férnandez y Morales, 2013).Así también, la definición de las tareas que involucró cada sprint al igual que las reuniones que se llevaron a cabo diariamente; permitieron la culminación exitosa del desarrollo del mismo; puesto que se revisaron los avances de cumplimiento y comprobación del correcto funcionamiento del mismo.

De acuerdo a lo expuesto anteriormente por diferentes autores, todos los casos de estudio mencionados anteriormente, concuerdan en los beneficios que brinda la aplicación de sistemas de información en las organizaciones; especialmente en el manejo de la información, optimización de procesos internos, mejoras en rendimientos financieros y relaciones con el cliente. Por eso, se afirma que el impacto de la implementación del presente proyecto es positivo ya que se cuenta con un sistema independiente al que posee actualmente el restaurante para sus ventas, lo cual no afectará en la operatividad diaria del negocio. El sistema que desarrollado brindará una opción en la cual se podrá cargar las ventas realizadas, y por medio de un proceso automático rebajará los insumos que se encuentre en el inventario de producción.

Por lo tanto, la implementación del 
sistema permitió que el restaurante mejore el control de los insumos de sus locales y así, el incremento de un margen de utilidad global; sugiriendo un precio estimado de venta de acuerdo al costo total de las recetas. El desarrollo del sistema en una plataforma web, se facilitó la visualización del stock global de los inventarios, contrastando al sistema actual, en el cual se generaba la información de cada local por separado para posteriormente consolidarla de una forma manual.

Este trabajo estuvo limitado en la actualización automática de las ventas generadas por el restaurante, ya que la emisión de las facturas era realizada por el sistema de un tercero. Como trabajo futuro se puede realizar la implementación de módulos complementarios como el mencionado anteriormente sistema de facturación; así como también, contemplar la migración del presente aplicativo a la nube a fin de optimizar costos de mantenimiento, seguridades, entre otros.

\section{Referencias}

Alfonso, P., Mariño, S. y Godoy, M. (2011). Propuesta metodológica para la gestión de proyecto de software ágil basado en la web. Multiciencias, 1(4), 395-401

Andreu, R., Ricart, J. E. y Valor, J. (1991). Sistemas de información y la organización: ventajas o desventajas competitivas?. Documentto Investigación $\mathrm{n}^{\circ} 203$. Universidad de Navarra. Madrid, España. Obtenido de:

http://www.iese.edu/research/pdfs/DI-02 03.pdf
Arbeláez, O., Medina, F. y Chaves, J. (2011). Herramientas para el desarrollo rápido de aplicaciones web. Scientia Et Technica, 17, 254-258.

Armbrust, M., Fox, A., Griffith, R., Joseph, A., Katz, R., Konwinski, A. y Zaharia, M. (2010). A view of cloud computing. Communications of the ACM,53, 50-58.

Aruquipa, M. (2012). Incertidumbres frente al Cloud Computing. Revista de Información, Tecnología y Sociedad, 7, 14-15.

Asociación Española de Contabilidad y Administración de Empresas. (2013). Sistemas de Información Integrados (ERP). Documentos AECA. Serie Nuevas Tecnologías y Contabilidad. Documento $\mathrm{n}^{\circ}$ 6. Madrid, España. Obtenido de: https://www.elmostrador.cl/media/2015/0 5/nt6.pdf

Avello, D., Pérez, B. L. y Gayo, J. (2001). Desarrollo del portal web de la EU de Ingeniería Tecnica en Informática de Oviedo. Actas de las VII Jornadas de Enseñanza universitaria de la Informática (págs. 39-44). Palma de Mallorca: AENUI. Obtenido de:

http://danigayo.info/publications/jenui20 01.pdf

Barrios, W., Godoy, M., Fernandez, M., Mariño, S., Ferreira, M. y Zarrabeitia, C. (2011). SCRUM: Experiencia de Aplicación en una Empresa de Desarrollo de Software del NEA. XVII Congreso Argentino de Ciencias de la Computación.

Bazán, P. (2008). X Workshopde Investigadores en Ciencias de la Computación. AJAX: un análisis tecnológico y posibilidades metodológicas (págs. 335-339). La Plata: Creative Commons.

Benvenuto, Á. (2006). Implementación de sistemas ERP, su impacto en la gestión de la empresa e integración con otras TIC. CAPIV Review, 4, 35-47. 
Blandón Andrade, J. y López Sotélo, J. (2012). BIO-ROUTE: Un simulador para redes de sensores inalámbricos. Educación en Ingeniería, 7(13), 26-27.

Centro de excelencia de software libre -CESLAM. (15 de Octubre de 2011). Implantación de un Sistema Integral de Gestión Empresarial (ERP) para un Distribuidor de Repuestos. Obtenido de:

https://www.bilib.es/fileadmin/descargas/ Implantacion_ERP_en_empresa.pdf

Chacón, G. (2007). La Contabilidad de costos, los sistemas de control de gestión y la rentabilidad empresarial. Actualidad Contable FACES, 10 (15), 29-45.

Colla, P. (2012). Marco para evaluar el valor en metodología SCRUM. 13th Argentine Symposium on Software Engineering, ASSE 2012 (págs. 32-46). Córdoba - Argentina: ESE - Instituto Universitario Aeronáutico

EDICOM. (2012). Edicom connecting business. Obtenido de:

https://www.edicomgroup.com/en_US/cu stomers/customers/unilever.html

Franco, R., Trejo, I. M. y Román, G. (2016). Cloud Computing: La utilización de un cuaderno electrónico de apuntes con tecnología de cómputo en la nube, como apoyo al procesode enseñanzaaprendizaje. En Aplicación del Saber: Casos y Experiencias (Vol. 2, págs. 3648 - 2653). Tabasco: Academia Journals.

García Doval, F. (2005). El papel de los portafolios electronicos en la enseñanza - aprendizaje de lenguas. Glosas didácticas: revista electrónica internacional, 14, 112-119.

García, D., Marín, S. y Martinez, F. (2006). La contabilidad de costos y rentabilidad en la Pyme. Contaduría y Administración, 218, 39-59

García-Holgado, A. y García-Peñalvo, F. (2015). Estudio sobre la evolución de las soluciones tecnológicas para dar soporte a la Gestión de la información. Informe Técnico GRIAL -TR-2015-001. Grupo de Investigación en Inter Acción y eLearning (GRIAL). Universidad de Salamanca. Obtenido de:

https:/gredos.usal.es/jspui/handle/10366/ 125415

Garrett, J. J. (18 de Febrero de 2005). Ajax: A New Approach to Web Applications. Obtenido de:

http://www.adaptivepath.com/publication s/essays/archives/000385.php

González, J. y Domínguez, J. (2012). Sistema integral automatizado de control de costos de construcción para empresas medianas. Ingeniería de Construcción, $18,15-32$.

Gutiérrez, V. y Vidal, C. J. (2008). Modelos de Gestión de Inventarios en Cadenas de Abastecimiento: Revisión de la Literatura. Inventory Management Models in Supply Chains: A Literature Review. Revista Facultad de Ingeniería de Antioquia, 43, 134-149.

Huiza Yampasi, E. (2012). Scrum Distribuido una metodología de desarrollo en la nube. Revista de Información, Tecnología y Sociedad, 77, 77-79.

Lavolpe, A. (2005). Los sistemas de costos y la contabilidad de gestión: Pasado presente y futuro. Costo y Gestión, 55, 661-672.

López, C. A. (2011). MApache, estilo de programación para el desarrollo rápido de aplicaciones ingenieriles orientadas a la web. Revista Inventum, 6 (10), 38-44.

Losavio, E., Matteo, A. y Rahamut, R. (2006). The 14th International Conference on Distributed Multimedia Systems. Caracterización de aplicaciones con servicios basados en calidad. (págs. 90-95). Boston: Knowledge Systems Institute Graduate School. 
Marcos, E., De Castro, V. y Vela , B. (2004). Una extensión de UML para el modelado de servicios web. e-Gnosis, 2, 1-13

Navarro, A., Férnandez, J. y Morales, J. (2013). Revisión de Metodologías ágiles para el desarrollo de software. Prospectiva, 11 (2), 30-39.

Nazareno, R., Leone, H. y Gonnet, S. M. (2013). In XVIII Congreso Argentino de Ciencias de la Computación. Trazabilidad de procesos ágiles: un modelo para la trazabilidad de procesos Scrum. La Plata: Creative Commons

Orjuela, A. y Rojas, M. (2008). Las metodologías de desarrollo ágil como una oportunidad para la Ingenieria del software educativo. Avances en Sistemas e Informática, 5 (2), 159-171

Osorio, C. (2008). Modelos para control de inventarios en las pymes Panorama, 2 (6), 4-10.

Rossetti, G. y Arcusín, L. (2013). Optimización del sistema de inventario en una empresa productora y comercializadora de helados. VII Congreso Argentino de Ingeniería Química. Rosario, Argentina. Obtenido de:

http://www.aaiq.org.ar/SCongresos/docs/ 04_025/papers/11a/11a_1510_069.pdf

Sánchez, M., Vargas, M., Reyes, B. y Vidal, O. (2011). Sistemas de Información para el control de inventarios del almacén del ITS. ConCiencia Tecnológica, 41, 41-46.

Santamaría, F., Ballesteros, J. y González, J. (2015). Plataforma cloud computing como infraestructura tecnológica para laboratorios virtuales, remotos y adaptivos. Revista Científica, 23, 98-110

Solmicro. (2011). Caso éxito con Solmicro ERP Industrial: Garsán. Obtenido de: https://www.solmicro.com/casos-de-exit o-testimoniales/casos-de-exito-erp-indust rial-garsan
Trasobares, A. H. (2003). Los sistemas de información: evolución y desarrollo.Proyecto social. Revista de relaciones laborales, 10-11, 149-165.

Yazyi, S. A. (12 de Junio de 2011). Una experiencia práctica de Scrum a través del aprendizaje basado en proyectos mediado por TIC en un equipo distribuido. Obtenido de: https://gredos.usal.es/jspui/bitstream/103 66/100082/1/TFM_YazyiSergio_Master. pdf 
